SUBJECT AREAS:

APPLIED PHYSICS

CHEMICAL PHYSICS

Received

17 December 2013

Accepted

7 April 2014

Published

2 May 2014

Correspondence and requests for materials should be addressed to F.J. (fiinustc@ustc.edu. cn) or T.N. (tongai@ cuhk.edu.hk)

* These authors contributed equally to this work.

\title{
Charging and discharging of single colloidal particles at oil/water interfaces
}

\author{
Peng $\mathrm{Gao}^{2 *}$, XiaoChen $\mathrm{Xing}^{5 *}$, Ye Li ${ }^{4}$, To Ngai ${ }^{5}$ \& Fan Jin ${ }^{1,2,3}$
}

'Hefei National Laboratory for Physical Sciences at Microscale, University of Science and Technology of China, Hefei, P. R. China 230026, ${ }^{2}$ Department of Polymer Science and Engineering, University of Science and Technology of China, Hefei, P. R. China $230026,{ }^{3}$ CAS Key Laboratory of Soft Matter Chemistry, University of Science and Technology of China, Hefei, P. R. China 230026, ${ }^{4}$ Department of Modern Physics, University of Science and Technology of China, Hefei, P. R. China 230026, ${ }^{5}$ Department of Chemistry, The Chinese University of Hong Kong, Shatin, N.T., Hong Kong.

The physical behavior of solid colloids trapped at a fluid-fluid interface remains in itself an open fundamental issue. Here, we show that the gradients of surface tension can induce particles to jet towards the oil/water interface with velocities as high as $\approx 60 \mathrm{~mm} / \mathrm{s}$ when particle suspensions come in contact with the interface. We hypothesize that rubbing between the particles and oil lead to the spontaneous accumulation of negative charges on the hemisphere of those interfacial particles that contact the oil phase by means of triboelectrification. The charging process is highly dependent on the sliding distances, and gives rise to long-ranged repulsions that protect interfacial particles from coagulating at the interface by the presence of electrolyte. These triboelectric charges, however, are compensated within several hours, which affect the stability of interfacial particles. Importantly, by charging different kinds of colloidal particles using various spreading solvents and dispersion methods, we have demonstrated that charging and discharging of single colloidal particles at oil/water interfaces impacts a broad range of dynamical behavior.

olid particles adsorbed at fluid-fluid interfaces have been traditionally exploited in emulsification ${ }^{1,2}$, mineral and crude oil recovery as well as in food industry ${ }^{3,4}$. More recently they were found in a rapidly increasing range of cutting-edge applications ${ }^{5-7}$, including the creation of nanostructured membrane for filtration and biphasic catalysis ${ }^{8-10}$, and the fabrication of nanocomposites with tunable electrical or optical properties ${ }^{11-13}$. For the purpose of supporting these emerging applications, an improved understating of interfacial behavior of particles $^{14-16}$ and their dynamics at a fluid interface ${ }^{17,18}$ is a key issue. Many earlier experiments ${ }^{19-22}$ were carried out on flat, planer fluid interfaces and various forces acting between the particles trapped at interface were proposed. However, to date, the behavior of solid particles at interfaces is still a very dynamic area of research with many open challenges that need to be met. For instance, it has been known that colloidal particles trapped at interface between water and a medium of low relative permittivity (air or oil) would form interfacial dipoles because of asymmetrical dissociation of surface charges around the particle ${ }^{17}$, which give arise to a long-ranged repulsion between particles. It is thereby expected that adding salts can screen these interfacial dipoles and repress the repulsion. However, Aveyard et al..$^{23,24}$ showed that charged colloidal particles produce extremely stable monolayers at an octane/water interface even at high electrolyte concentration, which cannot be addressed by dipole-dipole repulsions alone. They proposed that this repulsive force lies in charge-charge repulsion, likely arising from a small fraction of dissociated (residual) charges trapped by tiny surface water droplets in the pores of the particle's rough surfaces and in the oil phase, which result in unscreened charge-charge repulsion. In this model, the presence of tiny water droplets in the oil phase is essential because these polar droplets can provide high dielectric "nests" for trapping charged ions and subsequently causing the repulsion. However, we argued that the proposed existence of these water droplets may not account for the presence of residual charges at a nonpolar oil, especially one that has a low dielectric constant $\left(\varepsilon_{o i l}<4.0\right)$, such as octane $\left(\varepsilon_{o i l}=2.0\right)$.

All the points put forth above provide us a standing point to revisit this unresolved problem. By using various state-of-the-art microscopy techniques, here we show that the residual charges do exist in the hemisphere of particles immersed in nonpolar oil (octane) and dominate the long-ranged repulsions between interfacial particles when the typical dipole-dipole repulsions are screened by added electrolytes. In addition, our results explicitly indicate that these residual charges are actually unstable, and are compensated in several hours, which could in turn attenuate the strength of the charge-charge repulsions and eventually cause particles aggregation at oil/water interfaces. Furthermore, we demonstrate that these unstable charges can be built spontaneously, especially common approach relies heavily on using a spreading solvent to facilitate dispersion of particles at 
the oil/water interface. Importantly, we find that charging or discharging of colloidal particles at oil/water interfaces is a general phenomenon, that thus provides an novel insight in understanding their interactions and assembly at the interfaces, including the significant heterogeneity of interfacial interactions that have been observed between particle pairs trapped at fluid interfaces ${ }^{25,26}$.

\section{Results}

Time evolution of colloidal particles trapped at oil/water interfaces. To our best knowledge, direct measurements of the interactions between colloidal particles trapped at oil/water interfaces, for example, by microscopy techniques, often encounter two technical problems: 1) Slow drifting of interfacial particles with a certain direction along the interface, and therefore the prolonged perturbation that alter the original spatial distribution of particles is expected to occur; 2) Introducing the spreading solvents, such as methanol or isopropanol, has been demonstrated to affect greatly the particle properties at the fluid interfaces ${ }^{27}$. Here, we first produce a flat and horizontal oil/water interface in a customized microscopic cell that can be double sealed (Fig. S1 and Fig. S3), thereby inhibiting any possible evaporation of oil or water that could cause particle drift at the interface; Secondly, unlike common dispersion approach, we directly dispersed microspheres at the interface using microinjection (Fig. S2), in which the quantity of spreading solvent can be reduced to as low as $5 \times 10^{-6}(\mathrm{v}: \mathrm{v})$ in the system. These two experimental improvements enable us to produce a highly stable and clean oil/water interface, allowing the long-term ( $>36$ hours) measurements that are essential to resolve precisely weak interactions between interfacial particles.

Next, we monitored time evolution of monodispersed polystyrene microspheres $(1.0 \mu \mathrm{m})$ trapped at an octane $/ \mathrm{H}_{2} \mathrm{O}$ interface in absence or presence of $\mathrm{NaCl}$ in the water phase over 10 hours.

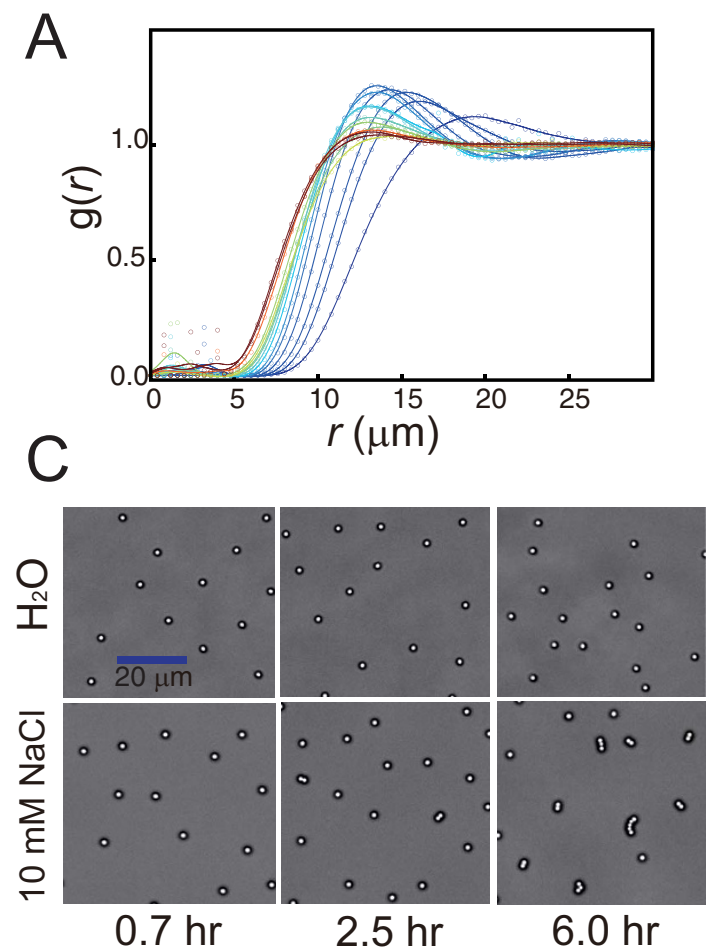

Surprisingly, we found that at the octane $/ \mathrm{H}_{2} \mathrm{O}$ interface, the particles initially were well separated at the interface, but the tendency for interfacial particles accessing to their neighbors increased gradually within the first 6 hours and then reached a stable state; this can be quantitatively characterized by the time evolution of radius distribution functions $(g(r))$ of interfacial particles ${ }^{28}$, as shown in Figure $1 a$. The time-dependent feature of $g(r)$ implies that interactions among interfacial particles, actually, are varying slowly. Similar phenomena have also been observed at an octane $/ 10 \mathrm{mM} \mathrm{NaCl}$ interface, as shown in Figure $1 b$, indicating that the time-dependent feature of $g(r)$ is inherent at oil/water interfaces, and is independent of whether presence of salt in water or not. In contrast to the microspheres trapped at an octane $/ \mathrm{H}_{2} \mathrm{O}$ interface, we find that those microspheres form small clusters at octane/10 $\mathrm{mM} \mathrm{NaCl}$ interface after 2 hours (Fig. 1c), which lead to appearing peaks in their $g(r)$, as shown in Figure $1 B$. Our results indicate that microspheres would become unstable at oil/water interfaces over time if the interfacial dipoles are screened. To further validate that, we conducted a series of experiments with the presence of different amounts $\mathrm{NaCl}$ in the water phase ranging from 0.01 to $10 \mathrm{mM}$. In all tested conditions, we consistently found that $g(r)$ always evolved over time, i.e., increasing the tendency of interfacial particles to access their neighbors, while the presence of sufficient salts in the water phase would greatly affect the stability of interracial particles and eventually form colloidal aggregates at interfaces, as shown in Figure $1 d$.

Attenuation of repulsions at oil/water interfaces. We have clearly shown that the $g(r)$ of interfacial particles possess a time-dependent feature at octane/water interfaces either in absence or presence of a monovalent salt in the water phase. Assuming that the system stays at quasi-equilibrium state in the every fast observing time (see the methods), we can quantitatively evaluate the pair potentials $(U(r))$ of interfacial particles at an octane $/ \mathrm{H}_{2} \mathrm{O}$ or octane $/ \mathrm{NaCl}$ interface by
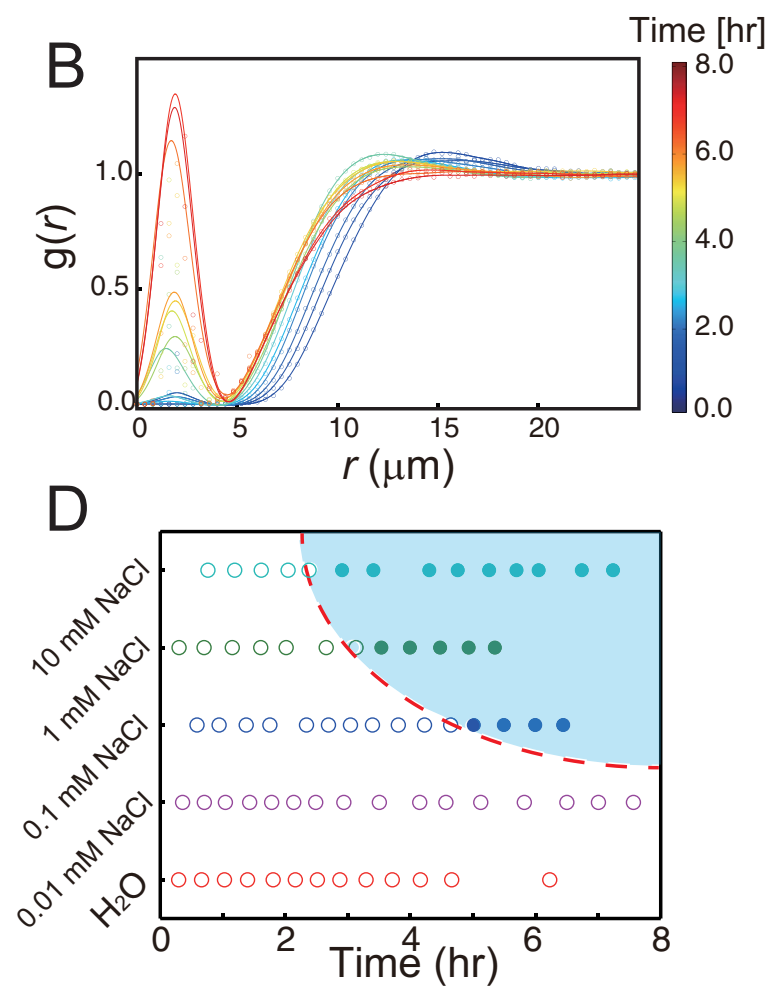

Figure 1 Time evolution of single colloidal particles trapping at oil/water interfaces in absence or presence of a monovalent salt (NaCl) in the water phase. Time evolutions of radius distribution functions $(g(r))$ of polystyrene microspheres $(1.0 \mu m)$ trapping at an $(a)$ octane/ $\mathrm{H}_{2} \mathrm{O}$ interface or $(b)$ an octane/10 mM NaCl interface as well as their bright-field images $(c)$, where $5 \%$ isopropanol was used as the spreading solvent, colors represent time lapse. (d) Stability of polystyrene microspheres trapping at oil/water interfaces with presence of different amounts $\mathrm{NaCl}$ in the water phase ranging from 0.01 to $10 \mathrm{mM}$, where solid symbols in the shadow represent emerging of colloidal aggregates at oil/water interfaces. 
their $g(r)$ using hypernetted chain (HCN) approximation, which was proved to be a better choice to resolve the long-ranged potentials ${ }^{28}$. Note that in the case of octane $/ 10 \mathrm{mM} \mathrm{NaCl}$ interface, we evaluate $U(r)$ of the trapped microspheres before the emerging of colloidal aggregates, which ensure that the system stays at quasi-equilibrium state. Figure $2 a$ and $2 b$ clearly indicated that 1 ) exclusive repulsions are detected at the octane $/ \mathrm{H}_{2} \mathrm{O}$ or the octane $/ 10 \mathrm{mM} \mathrm{NaCl}$ interface, in which $U(r)$ monotonically decay with increasing separation of microspheres; 2) The magnitude of evaluated $U(r)$ were attenuated gradually over time, which is independent of whether presence of $\mathrm{NaCl}$ in water. It is desirable to note that the long-ranged attractions, which likely arise from capillary forces ${ }^{19,29}$ and have been often observed among the microspheres trapped at air/water interface ${ }^{30,31}$, have not been detected in our experiments.

A number of factors can in principle lead to the time-dependence of $U(r)$, including 1 ) the gradual change of particle wettability stemming from physical aging of contact line $\mathrm{e}^{22} ; 2$ ) the local deformation of interfaces attributed by electrodipping forces ${ }^{32}$ and 3 ) the spreading solvent (isopropanol) may swell these microspheres. To address these factors, a high-resolution confocal microscope was used to investigate the microstates of these microspheres trapped at a vertical octane/water interface. Confocal images clearly show that i) three phase contact angles $(\theta)$ of interfacial particles were nearly independent of time either at octane $/ \mathrm{H}_{2} \mathrm{O}$ or at octane $/ 10 \mathrm{mM} \mathrm{NaCl}$ interface (Figs. $2 c$ and $2 d$ ), which thereby cannot be responsible for the attenuation of $U(r)$; ii) No significant local deformations have been observed at interfaces (Fig. $2 d$ ), which may explain why we did not detect long-ranged attractions among these microspheres; iii) No significant swelling of those interfacial particles have been observed. To further address this issue, we dispersed those microspheres at octane $/ \mathrm{H}_{2} \mathrm{O}$ or at octane $/ 10 \mathrm{mM} \mathrm{NaCl}$ interface by using methanol as the spreading solvent because polystyrene is unlikely to be dissolved in it. We find that using methanol as alternative spreading solvent also lead to the time-dependent feature of $g(r)$ or $U(r)$ either at octane $/ \mathrm{H}_{2} \mathrm{O}$ or at octane $/ 10 \mathrm{mM} \mathrm{NaCl}$ interface, which are similar to that by using isopropanol as the spreading solvent (Fig. S4), suggesting that the swelling effect cannot be responsible for the attenuation of $U(r)$. Moreover, we noticed $\theta=116^{\circ} \pm 10$ at octane $/ 10 \mathrm{mM}$ $\mathrm{NaCl}$, which was slightly larger than $\theta=100^{\circ} \pm 7$ measured at octane $/ \mathrm{H}_{2} \mathrm{O}$ interface. This difference may result from the surface charges locating at hemisphere immersed in the water phase because their image charges have same sign that would push interfacial particles toward water phase. In this way, screening these surface charges by adding salts would lead to more proportion of microspheres residing in the oil phase and less amount protruding into the aqueous phase $\mathrm{e}^{10,33}$.

Discharging of interfacial particles at oil/water interfaces. We next ask whether the attenuation of residual charges can be responsible for the attenuation of $U(r)$. First, we neutralized the charges that were dissociated from the surface of carboxylate-modified microspheres trapped at octane $/ \mathrm{H}_{2} \mathrm{O}$ interface by adjusting $\mathrm{pH}$ value in water to 4.85 , as indicated by the Zeta potential $(\xi)$ of these particles approaching zero $(\xi=-1 \mathrm{mV} \pm 3)$ in the bulk condition $(\mathrm{pH}=$ 4.85); second, we utilized microelectrophoresis (Fig. S3c) to monitor the electrophoretic mobility of these interfacial particles. By carrying out theoretical calculation, we find that our microelectrophoresis device can generate the static electric filed as high as 2500 to $5200 \mathrm{~V} / \mathrm{m}$ near the interface when a $60 \mathrm{~V}$ DC was applied, as shown in Figure S5, which can push the interfacial particles that carried around $100 e$ charges toward the anode with a velocity ranging from 5 to $11 \mu \mathrm{m} / \mathrm{s}$. In our experiments, we find that initially the particles always migrated to anode when a $60 \mathrm{~V} \mathrm{DC}$ was applied (Mov. S1) with an average velocity of $3 \mu \mathrm{m} / \mathrm{s}$, but no
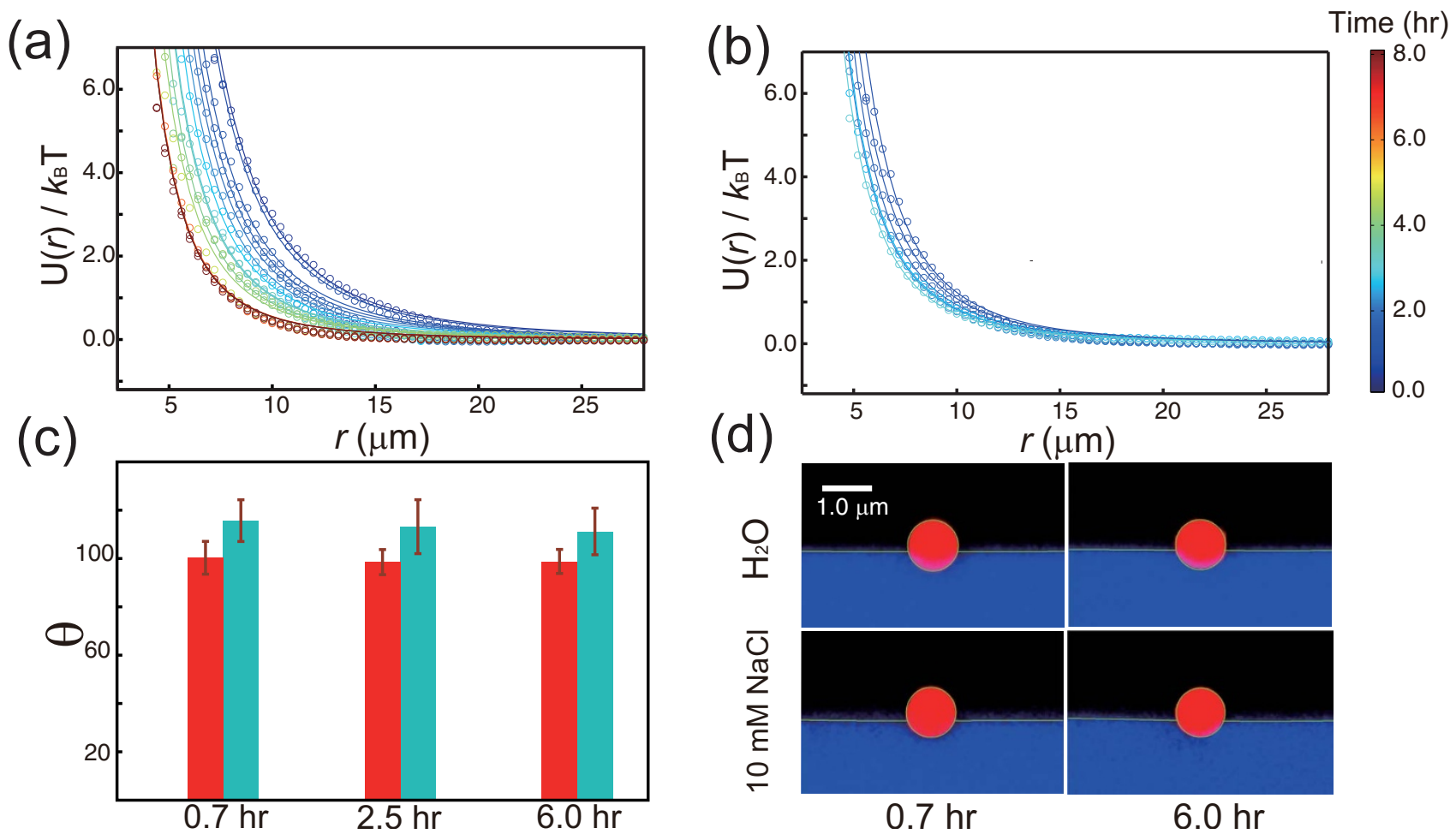

Figure $2 \mid$ Attenuation of repulsions among single colloidal particles at oil/water interfaces in absence or presence of $\mathrm{NaCl}$ in the water phase. Time evolutions of pair potentials $(U(r))$ of polystyrene microspheres trapping at an $(a)$ octane $/ \mathrm{H}_{2} \mathrm{O}$ interface or $(b)$ an octane/10 mM NaCl interface, where symbols or lines represent $U(r)$ or their fitting curves based on equation (1) and (2), colors represent time lapse. (c) Time dependence of three phase contact angles $(\theta)$ of polystyrene microspheres trapping at an octane $/ \mathrm{H}_{2} \mathrm{O}$ (red bars) or at an octane $/ 10 \mathrm{mM} \mathrm{NaCl}$ (cyan bars) interfaces, and their confocal images $(d)$, where blue colors or green lines respectively represent emissions of coumarin in aqueous phase or boundaries determined by finding maximum gradients of intensities. 
(a)

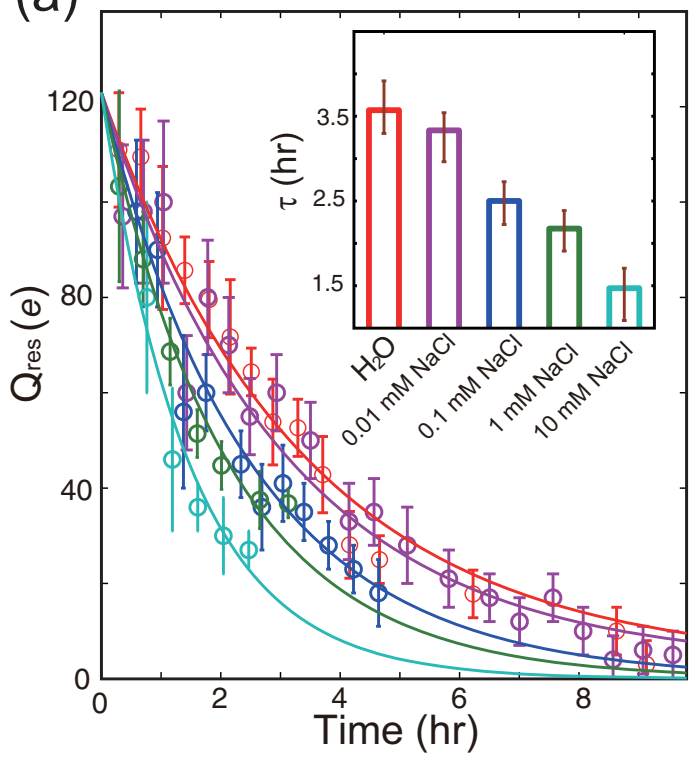

(b)

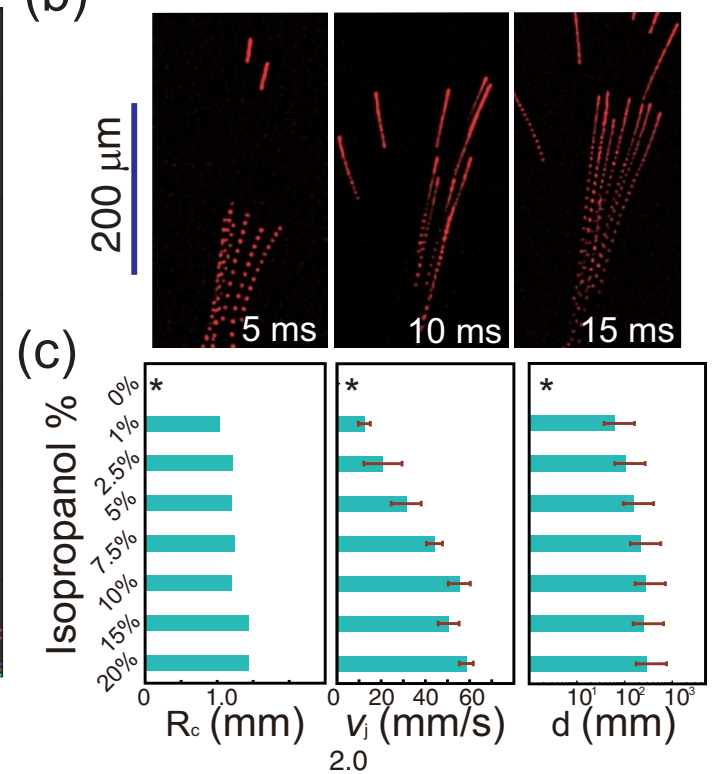

Figure 3 Discharging and spreading of single colloidal particles at oil/water interfaces. (a) Fading of residual charges on polystyrene microspheres trapping at oil/water interfaces in presence of different amounts $\mathrm{NaCl}$ in the water phase, where solid lines represent their fitting by single exponentially decay, and inset of $(a)$ shows their characteristic decay time $(\tau)$. Dispersion of particle suspensions containing different amounts of isopropanol ranging from 1 to $20 \%$ at an octane/ $\mathrm{H}_{2} \mathrm{O}$ interface: (b) Spinning-disk confocal images acquired with a certain exposure time ranging from 5 to 20 ms (containing $2.5 \%$ isopropanol in the suspension), $(c)$ sliding velocities $\left(v_{j}\right)$ or radius convection zone $\left(R_{c}\right)$ or sliding distance $(d)$, where the symbol $(*)$ presents that no jets had been observed at the interface.

significant electrophoretic mobility was observed after 4 hours, demonstrating that the residual charges fade out in several hours.

To quantify the discharging of interfacial particles, we fitted the repulsive potentials measured at the octane $/ 10 \mathrm{mM} \mathrm{NaCl}$ interfaces using a theoretically pair potential $\left(U_{r e s}(r)\right)$ that accounts for a point charge $\left(Q_{\text {res }}\right)$ located in the oil phase at a distance $(a)^{23}$,

$$
\frac{U_{r e s}(r)}{k_{B} T} \simeq 4 \pi e^{-2} Q_{r e s}^{2}\left(\frac{l_{B}^{o}}{r}-\frac{l_{B}^{o}}{\sqrt{4 a^{2}+r^{2}}}\right)
$$

where $k_{B}$ is the Boltzmann constant, $T$ is the absolute temperature, $l_{B}^{o}=e^{2} / 4 \pi \varepsilon_{o i l} \varepsilon_{0} k_{B} T$ is the Bjerrum length in octane, $e$ is the elementary charge, and $\varepsilon_{0}$ is the vacuum permittivity. Figure $2 B$ indicated that equation (1) can fit the experimental data perfectly, further demonstrating that these long-ranged repulsions found at octane/ $10 \mathrm{mM} \mathrm{NaCl}$ interface is a direct result from electrostatic interactions and can be solely attributed to the residual charges.

Importantly, the quantity of residual charges decreases gradually over time, as shown in Figure $3 a$. In addition to residual charges leading to the charge-charge repulsion acting through unscreened oil phase, the interfacial dipoles would provide stable repulsions $\left(U_{\text {dip }}(r)\right)$ at the octane $/ \mathrm{H}_{2} \mathrm{O}$ interface, which can be theoretically described as $^{20}$

$$
\frac{U_{d i p}(r)}{k_{B} T} \simeq 8 \pi e^{-2} Q_{d i p}^{2}\left(\frac{l_{B}^{w}}{r}\right)\left(\frac{\lambda_{D}}{r}\right)^{2}
$$

where $Q_{d i p}$ is the electric quantities of dissociated surface charges, $l_{B}^{w}$ is the Bjerrum length in water, and $\lambda_{D}$ is the Debye screening length. To evaluate $Q_{\text {res }}$ at octane $/ \mathrm{H}_{2} \mathrm{O}$ interfaces, equation (2) is used to first fit the experimental data obtained after 8 hours to find the $U_{d i p}(r)$, subsequently equation (1) is used to resolve the $Q_{\text {res }}$ by excluding $U_{\text {dip }}(r)$ in the original data. The corresponding fitting curves are shown in Figure $2 a$. Similar to the octane $/ 10 \mathrm{mM} \mathrm{NaCl}$ interface, we found that $Q_{\text {res }}$ decayed monotonically over time, but their discharging is slower than that found for octane $/ 10 \mathrm{mM} \mathrm{NaCl}$
(Fig. 3a). To gain insight into the discharging effect, we carried out a series of experiments with the presence of different amount $\mathrm{NaCl}$ in water. In all tested conditions, we found that $Q_{\text {res }}$ always decayed over time (Fig. 3a), and can be fitted with a single exponential decay $Q_{r e s}^{0} \exp (-t / \tau)$, where $Q_{r e s}^{0}$ is the $Q_{\text {res }}$ at $t \rightarrow 0, \tau$ is the characteristic decay time. Inset of Figure $3 a$ clearly indicated that increasing ionic strength in water phase would lead to much faster discharging of microspheres at the oil/water interface, suggesting that the aqueous phase provides a discharging path for the interfacial particles.

Spreading of particles at oil/water interfaces. It is reasonable to anticipate that these charges are generated when the microspheres arrive at the oil/water interface, giving the fact that microspheres or their suspensions could not carry any unstable charges before the dispersion. Therefore, a high-speed spinning-disk confocal microscope was applied to monitor the dispersion of fluorescent microspheres at an octane $/ \mathrm{H}_{2} \mathrm{O}$ interface, which ensures that only interfacial particles can been seen. Surprisingly, we observed that these microspheres jet along the interface with extremely high speeds $(20-60 \mathrm{~mm} / \mathrm{s})$ at the moment of contact with the interface (Mov. S2). The sliding velocity $\left(v_{j}\right)$ were estimated by measuring the travel distances of the corresponding flight paths in images that were captured by an EMCCD camera with specific exposure times, as shown in Figure $3 b$. Note that the interfacial jet is not a result of microinjection due to the fact that particles injected to either $\mathrm{H}_{2} \mathrm{O}$ or octane do not jet. Our findings are similar to the case of dispersion of small particles at air/water interfaces that has been reported by Singh et.al, in which they ${ }^{34}$ proposed that the capillary force pull particles into the interface, causing them to accelerate to a high velocity. However, for the oil/water interface, we noticed that 1) trace amounts of spreading solvents in the suspensions, such as isopropanol, was essential to induce the interfacial jets; 2) $v_{j}$ was largely correlated with the quantity of spreading solvent, as shown in Figure $3 c$. These finding suggest that spreading solvents play a significant role in facilitating the spreading of particles at the interface; this is known as the Marangoni effect ${ }^{35}$. 
To further demonstrate that the Marangoni effect can lead jetting of particles at the interface, we captured the larger-scale images of these interfacial jets during the microinjections. We find that 1) the microinjection of isopropanol (aq.) at the interface can strongly induce the convections, as shown in Figure S6a, which is in a good agreement with the Marangoni effect that also can induce convection at the interface; 2$)$ the characteristic radius $\left(R_{c}\right)$ of the convection zone are insensitive with the quantity of isopropanol ranging from $2.5 \%-10 \%$, as shown in Figure $3 c$. Next, we estimated the sliding velocity of particles at the interface in different conditions by using a simple model (see the method) that only accounts the surface tension driven phenomena, as

$$
v_{j} \simeq \frac{\left[\gamma\left(c_{b}\right)-\gamma(0)\right] d_{\mathrm{p}}}{\eta R_{c}}
$$

where $\gamma\left(c_{b}\right)$ or $\gamma(0)$ is the surface tension of isopropanol (aq.)/octane or water/octane interface, $c_{b}$ is the concentration of the isopropanol in particles suspensions, $d_{p}$ is the particle size and $\eta$ is the interfacial viscosity. Figure $\mathrm{S} 6 b$ clearly indicated that the predicted sliding velocities $\left(v_{j}^{\prime}\right)$ agree well with the experimental observations (Fig. $3 c$ ) at the conditions of containing $2.5-10 \%$ isopropanol in the particle suspensions, demonstrating that jetting of particles at the interface mainly arose from the gradient of surface tensions.

Charging particles at oil/water interfaces. Previous findings indicated that the gradients of surface tension would drive particles rubbing to the interface, which inspire us to conjecture that fast rubbing may induce the electrons transfer from one insulator (octane) to another (polystyrene microspheres). If yes, the quantity of residual charges building at interfacial particles should highly depend on the dispersion processes. Thus, we utilized aforementioned methods to evaluate the $Q_{\text {res }}^{0}$ with distinctive dispersion conditions, including varying the isopropanol content in particles suspensions during the microinjection or directly pipetting particle suspensions to the interfaces (Fig. S7). Note that changing of isopropanol content from $1 \%$ to $20 \%$ in suspensions only slightly alters the wettability of particles at the interface (Fig. S8) because the isopropanol concentrations in our systems are very low ${ }^{27}$. Figure $4 a$ indicated that $Q_{\text {res }}^{0}$ are positively correlated to the content of isopropanol when the particles were injected to the interface, confirming that dispersion conditions strongly affect $Q_{\text {res }}^{0}$. Moreover, we find that the dispersion of particles by direct pipetting leads to less $Q_{\text {res }}^{0}$ (Fig. $4 b$ ) comparing to that by microinjections.

To further elucidate these observations, we quantified the triboelectrification effect by using two surface states theory (TSST). In the $\mathrm{TSST}^{36}$, rubbing one insulator to another can induce electrons transfer from one surface to the other, in which the direction of transfer depends on triboelectric series ${ }^{37}$; and the net charges can be quantified as (see the methods),

$$
Q_{\text {res }}^{0} \simeq Q_{\max }[1-\exp (-d / \lambda)]
$$

where $Q_{\max }$ is the maximum charge quantities, $d$ is the sliding distance and $\lambda$ is the characteristic distance. Equation (4) clearly indicated that $Q_{\text {res }}^{0}$ are positively correlated to the sliding distance rather than the sliding velocity. Therefore, we estimated the distances of particles sliding on the interface by measuring the time that particles stay at the convection zone because we have known the sliding velocity. We find that the time is ranged from 2 to $8 \mathrm{~s}$ during the microinjection, in which the characteristic time $\left(t_{c}\right)$ is around $\sim 5 \mathrm{~s}$ that is insensitive to the content of isopropanol, $d$ thus can be estimated by $v_{j} t_{c}$, as shown in Figure $3 c$. These results readily explained why $Q_{\text {res }}^{0}$ are positively correlated to the content of isopropanol in the particle suspensions (Fig. 4a). Most importantly, our results displayed that changing $d$ from 60 to $250 \mathrm{~mm}$ can strongly affect the triboelectrification, which suggests that $\lambda$ is in the range of $\sim 100 \mathrm{~mm}$ that agrees (a)
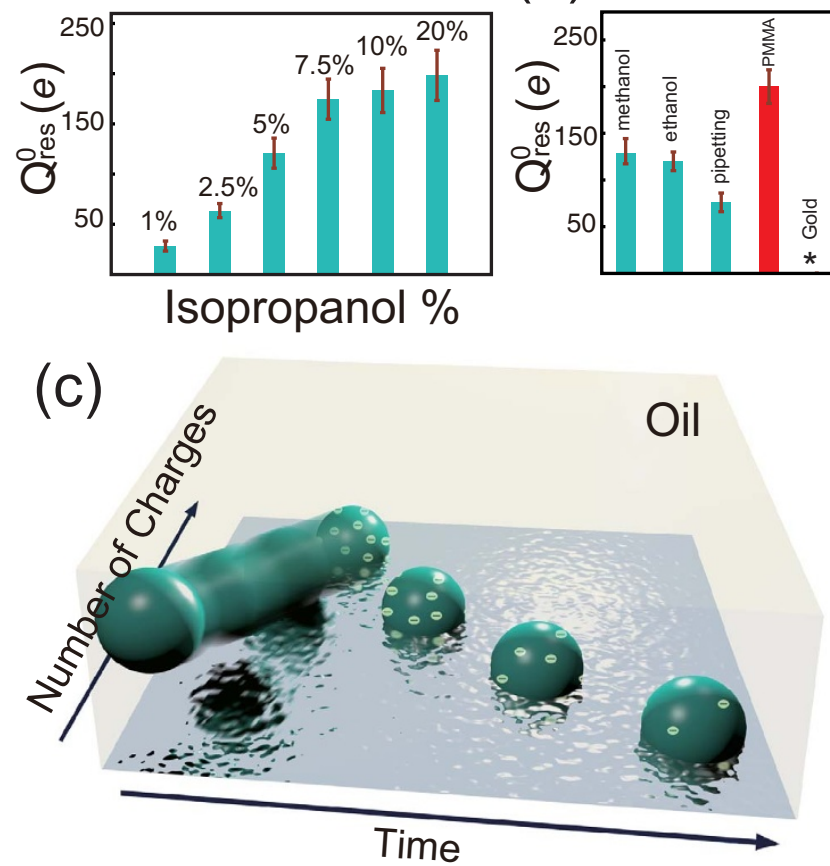

Figure $4 \mid$ Charging of single colloidal particles at oil/water interfaces. Charging polystyrene microspheres (cyan bars) at an octane/10 $\mathrm{mM} \mathrm{NaCl}$ interface using distinct suspensions: $(a)$ containing different amounts of isopropanol ranging from 1 to $20 \%,(b)$ containing $5 \%$ methanol or ethanol or using pipetting for dispersions. Charging polymethylmethacrylate (red bar) or gold microspheres at an octane/ $10 \mathrm{mM} \mathrm{NaCl}$ interface, where the symbol $\left({ }^{*}\right)$ presents that the long-ranged repulsion had not been observed. (c) Cartoon schematically shows charging and discharging of single colloidal particles at an oil/water interface.

well with Lowell's experimental results ${ }^{38}$. Moreover, we find that dispersion particles by pipetting lead particles much faster jetting on the interface $(160 \mathrm{~mm} / \mathrm{s})$ compared with the microinjection, however the interfacial flows can only be lasted for $0.3 \mathrm{~s}$. As the result, $d$ is around $\sim 50 \mathrm{~mm}$, which is well explained why dispersion particles by pipetting result in less $Q_{\text {res }}^{0}$ comparing to that by microinjection.

To further validate that these residual charges are transient charges, we dispersed gold particles at the oil/water interface in presence of $10 \mathrm{mM} \mathrm{NaCl}$, and we did not observe the long-ranged repulsions. This indicates that no residual charges exist in the hemisphere of gold particles immersed in oil, as shown in Figure $4 b$. We also investigated factors that affect the charging of particles at interfaces, by dispersing colloidal particles at oil/octane interfaces with the presence of $10 \mathrm{mM} \mathrm{NaCl}$ in water by using various experimental conditions, e.g.; using methanol or ethanol as spreading solvents or using polymethylmethacrylate (PMMA) microspheres. Figure $4 b$ clearly indicated that the value of $Q_{r e s}^{0}$ depends on experimental conditions. However, the charging of colloidal particles at oil/water interfaces is quite general.

\section{Discussion}

The key results extracted from the data presented above provide a new insight into interactions and assembly of solid particles at oil/ water interface. Moreover, our result can be used to explain some physical behaviors that are not well understood. For instance, Park et al. directly measured the repulsive forces between particle pairs trapped at oil/water interface using optical tweezers. They found that 
the repulsions actually are heterogonous ${ }^{26}$, highly depending on the individual pairs to be selected. In addition, the amount of sodium dodecyl sulfate (SDS) presence in the water phase can gradually attenuate the strength of this repulsion at the interface ${ }^{25}$. The finding of charging of single particles at interface can readily explain the heterogonous feature of these repulsive forces because these produced charges largely relate to the sliding distances and which are varied for individual particles, whereas attenuation of this repulsion over time are most likely related the discharging of these particles at interface.

In summary, we have shown that single colloidal particles can be spontaneously charged when spreading solvents are used to disperse them at oil/water interfaces. This is due to the gradients of surface tension driving particles sliding at the interface, which in turn lead to triboelectric charges transferring from oil to the colloidal particles. These residual charges dominate the long-ranged electrostatic repulsions, and prevent the interfacial particles against aggregation even when the conventional dipole-dipole repulsions are screened by adding monovalent salts in the aqueous phase. However, these triboelectric charges are unstable, and can fade out in several hours, thus gradually attenuating the repulsions among interfacial particles. As the interfacial particles are slowly discharged, they become unstable when their dipoles are screened. Charging and discharging of single colloidal particles at oil/water interfaces were graphically summarized in Figure $4 c$. Charging of colloids at oil/water interfaces plays remarkable roles in interfacial interactions of colloids, and our studies suggest that such phenomenon is general. It may prove to be a novel mechanism to induce transient charges on biological macromolecules, such as DNAs, proteins and viral particles at fluid interfaces.

\section{Methods}

Materials. The coverslips $(25 \times 25 \times 0.17 \mathrm{~mm}$, Fisher $)$ were pre-washed by sonication ( $5 \mathrm{~min}$ ) in acetone, ethanol and ultrapure water $(18.2 \mathrm{M} \Omega \cdot \mathrm{cm}$ Millipore), respectively. Afterward, they were rinsed with 5\% (aq.) hydrogen fluoride for $10 \mathrm{~s}$, and then washed with water immediately. The cleaned coverslips were finally dried with nitrogen and further treated $(5 \mathrm{~min})$ with a plasma cleaner before assembly on the microscopic sample cell. The resultant static contact angles of water on these surfaces almost approach to $0^{\circ}$. The hydrophobic modified coverslips were prepared by treating the cleaned coverslips with [(chloromethyl)phenylethyl]-

Trimethoxysilane (CTMS) in toluene. Carboxylate-modified fluorescent microspheres (polystyrene $1.0 \mu \mathrm{m}$ in diameter, Invitrogen) were purified by resuspending $2 \mu \mathrm{L}$ concentrated suspensions $(\sim 2 \%)$ in $1.5 \mathrm{~mL}$ ultrapure water, then washed by centrifugation to remove stabilizer. The stabilizer-free microspheres were finally dispersed in spreading mediums, containing different amounts (1 to $20 \%$ ) of isopropanol, methanol or ethanol in water, to a fixed volume fraction $\sim 1 \times 10^{-4}$, which were monitored by their optical densities at $600 \mathrm{~nm}\left(\right.$ O.D. $\left.{ }_{600}\right)$. The averaged surface charge density of the microsphere was around $1.3 \mu \mathrm{C} \cdot \mathrm{cm}^{-2}$, estimating by their Zeta potentials ( $\xi=-55 \mathrm{mV} \pm 3$ ) measured in a buffer containing of $10 \mathrm{mM}$ $\mathrm{NaCl}$. Note that none of cross-linked polystyrene microspheres may likely contain short PS chains that dissolve in the spreading mediums. Therefore, these PS microspheres were directly dissolved in the tetrahydrofuran (THF) to determine their molecular weight distribution. Next, their average molecular weight $\left(M_{n}=1.5 \times\right.$ $\left.10^{5} \mathrm{~g} / \mathrm{mol}, M_{w}=6.8 \times 10^{5} \mathrm{~g} / \mathrm{mol}\right)$ as well as polydispersity $(\mathrm{P} . \mathrm{DI}=4.2)$ were measured by using gel permeation chromatography (GPC). The GPC result indicated that these PS microspheres do not contain short PS chains, in which the molecular weight of smallest fraction is $\sim 1.0 \times 10^{4} \mathrm{~g} / \mathrm{mol}$. Polymethylmethacrylate (PMMA) microspheres $(1.0 \mu \mathrm{m}$ in diameter) were a gift from Xu's lab in The Chinese University of Hong Kong. Gold colloids (300 nm in diameter) were obtained from Xiong's Lab in University of Science and Technology of China. The ultrapure water or ultrapure octane (electronic grade, $>99.999 \%$, Sigma-Aldrich) was filtrated with a syringe filter (PTFE $0.2 \mu \mathrm{m}$ Millipore) to remove dusts before the preparation of octane $/ \mathrm{H}_{2} \mathrm{O}$ interfaces.

Preparation of oil/water interfaces and dispersion microspheres at interfaces. Horizontal octane $/ \mathrm{H}_{2} \mathrm{O}$ or octane $/ \mathrm{NaCl}$ interface was prepared in a customized microscopic cell, which was assembled with a coverslip, a PET spacer with thickness of $110 \mu \mathrm{m}$ and a cup-like glass cell, as shown in Figure S1 $a$. First, ultrapure water was gently added in bottom of cell to reach a height of $110 \mu \mathrm{m}$, and then $\sim 2.0 \mathrm{~mL}$ ultrapure octane was slowly added to form a horizontal octane $/ \mathrm{H}_{2} \mathrm{O}$ interface that was stuck with the PET spacer exactly (Fig. S1 $b$ ); Second, $0.2 \mu \mathrm{L}$ microsphere suspension, typically in $5 \%$ isopropanol (aq.), was directly microinjected at the interface with a flow rate of $0.1 \mu \mathrm{L} / \mathrm{min}$ using a microinjector (Fig. S2a) composing of a XYZ manipulator, a syringe pump and a glass capillary (15-30 $\mu \mathrm{m}$ in diameter), in which bright-field microscope (Olympus IX-81) with a $\times 10$ objective was used to monitor the microinjection (Fig. S2c); Octane/ $\mathrm{NaCl}$ interface was produced by further adding $\mathrm{NaCl}$ in water via microinjection (Fig. S2b), where concentration of $\mathrm{NaCl}$ was determined with the volume ratio of $\mathrm{NaCl}\left(100 \mathrm{mM}\right.$ aq.) and $\mathrm{H}_{2} \mathrm{O}$. Finally, the small chamber containing the octane $/ \mathrm{H}_{2} \mathrm{O}$ or octane/ $\mathrm{NaCl}$ interface was sealed with a $(20 \times$ $20 \times 1.1 \mathrm{~mm}$ ) glass slide and the whole microscopic cell was further sealed with a polystyrene lid, as shown in Figure $\mathrm{S} 3 \mathrm{~A}$. Vertical octane $/ \mathrm{H}_{2} \mathrm{O}$ or octane $/ \mathrm{NaCl}$ interface was prepared in a device, as shown in Figure $\mathrm{S} 3 b$, composing a $30 \mu \mathrm{m}$ PTFE spacer and two hydrophobic modified coverslips whose water contact angles approach to $\sim 90^{\circ}$. Microspheres were dispersion at the vertical oil/water interface via the same microinjection procedure described above.

Tracking of microspheres at horizontal oil/water interfaces. An Olympus IX81 inverted-microscope equipped with a $40 \times$ objective and a sCMOS camera (Andor Zyla), was used to acquire bright-field images of microspheres trapped at horizontal octane $/ \mathrm{H}_{2} \mathrm{O}$ or octane $/ \mathrm{NaCl}$ interface with a frame rate $0.5 \mathrm{fps}$. A typical dataset, usually containing 2400 images with a field size of $409 \times 346 \mu \mathrm{m}^{2}$, has recorded trajectories of single microspheres $\left(r_{i}(t)\right)$ at oil/water interface. The datasets were continuously collected every 20 minutes over 10 hours, starting from the beginning of dispersions. These experiments were repeated multiple times in each identical conditions, thus over 300 datasets ( $>720,000$ images) were acquired and analyzed in total, in which the $2560 \times 216016$-bit greyscale images were firstly converted to binary images for detection of microsphere with a standard image processing algorithm coding by MATLAB, and the $\mathrm{x}-\mathrm{y}$ positions of microsphere centroids were subsequently determined and linked individually over time by using a particle tracking algorithm ${ }^{39}$.

Measurements of radius distribution functions and pair potentials of microspheres at horizontal oil/water interfaces. The trajectories of single microspheres $\left(\mathrm{r}_{i}(t)\right)$ in each dataset was used to calculate the radius distribution function $(g(r))$ by time-averaging pair correlations $\left(\sum_{i \neq j} \delta\left(\mathrm{r}_{1}-\mathrm{r}_{i}\right) \delta\left(\mathrm{r}_{2}-\mathrm{r}_{j}\right) / n\right)$ in every moment over 20 minutes, where $r=\left|\mathrm{r}_{1}-\mathrm{r}_{2}\right|$ is the spatial separation and $n$ is the surface density of microspheres. The pair potentials $U(r)$ was numerically evaluated from $g(r)$ using the hypernetted chain ( $\mathrm{HCN}$ ) approximation, in which $U(r)=-k_{B} T$ $\ln [g(r)]+n k_{B} T I(r)$ was applied, where $k_{B}$ is the Boltzmann constant, $T$ is the absolute temperature, and convolution integral $I(r)=\left[g\left(r^{\prime}\right)-1-n I(r)\right]\left[g\left(\left|\mathrm{r}^{\prime}-\mathrm{r}\right|\right)-1\right] d^{2} r^{\prime}$ can be obtained iteratively, starting with $I(r)=0^{28}$. Note that $U(r)$ can be evaluated from $g(r)$ only when the system stay at the equilibrium state or at quasi- equilibrium state. In our case, the interfacial particles can be either at stable or at unstable state, which depends on time and whether existing sufficient salts in water phase (Fig. 1d). Therefore, only the $g(r)$ at the stable region can be used to evaluate $U(r)$ by assuming that the system stays at quasi- equilibrium state.

Microelectrophoresis. Two platinum-wire electrodes $(100 \mu \mathrm{m}$ in diameter $)$ with a $0.3 \mathrm{~mm}$ separation were placed in the octane, $\sim 110 \mu \mathrm{m}$ above from the horizontal oil/water interface, as shown in Figure S3c. A 60 V DC was applied to generate a static electric field along the interface, and bright-field microscope equipped with a $20 \times$ objective was used to monitor the electrophoretic mobility of microspheres (Mov. S1) trapped at a horizontal octane $/ \mathrm{H}_{2} \mathrm{O}$, where the $\mathrm{pH}$ of aqueous phase was adjusted to 4.85 by $\mathrm{HCl}$ (aq.). Note that, in this set up, the electric field near the interface will greatly reduce by the field that was induced by their image charges. To assess the effective electric field near the interface, we have carried out a theoretical calculation by using COMSOL, as shown in Figure S $5 a$. Our results indicate that the resultant electric field is ranged from 2500 to $5200 \mathrm{~V} / \mathrm{m}$, depending on the distance apart from the interface, as shown in Figure $\mathrm{S} 5 b$.

Measurements of contact angles of microspheres at vertical oil/water interfaces. $15 \mu \mathrm{M}$ coumarin was added in water for imaging of oil/water interface. Fluorescent microspheres trapped at vertical oil/water interface were examined with a confocal microscope (Olympus FV1000) equipped with a $100 \times$ oil objective, in which coumarin and microspheres were excited simultaneously with 405 and $543 \mathrm{~nm}$ lasers to acquire confocal images via two distinct emission channels (425-475 nm and 555$655 \mathrm{~nm}$ ). The boundaries of the oil/water interface and microspheres were respectively evaluated by finding the maximum intensity gradients from their images. Three phase contact angle $(\theta)$ of single interfacial particle was geometrical measured using their determinate boundaries. These experiments were repeated multiple times in each identical conditions, thus over 500 confocal images, typically containing $2 \sim 5$ interfacial particles, were acquired and analyzed in total, enabling us to actually evaluate the $\theta$ in each conditions by averaging twenty single results at least.

Observation of spreading of microspheres at horizontal oil/water interfaces. A spinning-disk confocal microscope (Andor Revolution) equipped with a $10 \times$ objective was used to monitor the spreading of fluorescent microspheres at horizontal oil/water interface using different dispersion methods, including microinjection (Mov. S2) of pipetting (Fig. S7). Fluorescent microspheres were excited with a $561 \mathrm{~nm}$ laser. High-speed confocal images were collected through an emission filter $(600 \pm$ $25 \mathrm{~nm}$ ). The dispersion speeds (sliding velocities) of microspheres at oil/water interface were assessed by measuring the length of their flowing light paths remained in images, capturing by an EMCCD (Andor iXon897) with a certain exposed time ranging from 1 to $20 \mathrm{~ms}$. 
Estimation the sliding velocity of particles at the interface. Marangoni effect produces a slip velocity in the tangential direction on a fluid-fluid interface when gradients in the surface are present ${ }^{40}$. As a result, the tangential force per unit area $\left(f_{s}\right)$ is given by ${ }^{41} f_{s}=\nabla_{s} \gamma$, where $\nabla_{s}$ denotes the surface tension $(\gamma)$ gradients. In one hand, the tangential force acting on the particles $\left(F_{s}\right)$ at interface is $F_{s} \simeq d_{p}^{2} \nabla_{s} \gamma$. On the other hand, the viscous force is $\simeq d_{p} \eta v_{j}$. Therefore, one can find $v_{j} \simeq d_{p} \nabla_{s} \gamma / \eta$. $\nabla_{s} \gamma$ can be further estimated by $\nabla_{s} \gamma \simeq\left[\gamma\left(c_{b}\right)-\gamma(0)\right] / R_{c}$ (for the microinjection) or by $\nabla_{s} \gamma \simeq\left[\gamma\left(c_{b}\right)-\gamma(0)\right] / r_{d}$ (for the pipetting), where $r_{d}$ is the radius of droplet. $\gamma\left(c_{b}\right)$ is expected to close to the surface tension of interface between the isopropanol aqueous solution and air $^{42}$

Quantification of triboelectrification. In the two state surface theory ${ }^{36}$, rubbing a spherical insulator to a insulate surface would induce net charges $(Q)$ on the spherical insulator: $Q=Q_{0} \lambda_{1}\left[1-\exp \left(-d / \lambda_{1}\right)\right]-Q_{0} \lambda_{2}\left[1-\exp \left(-d / \lambda_{2}\right)\right]$ (eq. 5), where $Q_{0}\left|\lambda_{1}-\lambda_{2}\right|$ is the maximum charges quantity, $\lambda_{1}$ or $\lambda_{2}$ are the characteristic distances for charges transfer into the lower and out of the upper states of the spherical insulator respectively. Note that the material of polystyrene locate at the rather negative positions in triboelectric series ${ }^{43}$, which suggests that charges transfer into the lower state is much easier than charges transfer out of the upper state; i.e., $\lambda_{1} \gg \lambda_{2}$. Therefore, equation (5) can be rewritten as equation (4), where $Q_{\max }=Q_{0} \lambda_{1}$.

1. Binks, B. P. Particles as surfactants - similarities and differences. Curr. Opin. Colloid Interface Sci. 7, 21-41 (2002).

2. Leal-Calderon, F. \& Schmitt, V. Solid-stabilized emulsions. Curr. Opin. Colloid Interface Sci. 13, 217-227 (2008).

3. Hunter, T. N., Pugh, R. J., Franks, G. V. \& Jameson, G. J. The role of particles in stabilising foams and emulsions. Adv. Colloid Interface Sci. 137, 57-81 (2008).

4. Dickinson, E. Food emulsions and foams: Stabilization by particles. Curr. Opin. Colloid Interface Sci. 15, 40-49 (2010).

5. Bos, M. A. \& van Vliet, T. Interfacial rheological properties of adsorbed protein layers and surfactants: a review. Adv. Colloid Interface Sci. 91, 437-471 (2001).

6. Krafft, M. P. \& Riess, J. G. Highly fluorinated amphiphiles and colloidal systems, and their applications in the biomedical field. A contribution. Biochimie. 80, 489-514 (1998).

7. Herzig, E. M., White, K. A., Schofield, A. B., Poon, W. C. K. \& Clegg, P. S. Bicontinuous emulsions stabilized solely by colloidal particles. Nat. Mater. 6 , 966-971 (2007).

8. Verma, A. et al. Surface-structure-regulated cell-membrane penetration by monolayer-protected nanoparticles. Nat. Mater. 7, 588-595 (2008).

9. Verma, A. \& Stellacci, F. Effect of surface properties on nanoparticle-cell interactions. Small 6, 12-21 (2010).

10. Leunissen, M. E., van Blaaderen, A., Hollingsworth, A. D., Sullivan, M. T. \& Chaikin, P. M. Electrostatics at the oil-water interface, stability, and order in emulsions and colloids. Proc. Natl. Acad. Sci. USA 104, 2585-2590 (2007).

11. Bausch, A. R. et al. Grain boundary scars and spherical crystallography. Science 299, 1716-1718 (2003)

12. Law, A. D., Buzza, D. M. A. \& Horozov, T. S. Two-dimensional colloidal alloys. Phys. Rev. Lett. 106, (2011).

13. Tavacoli, J. W., Thijssen, J. H. J. \& Clegg, P. S. Particle-stabilized oscillating diver: a self-assembled responsive capsule. Soft Matter 7, 7969-7972 (2011).

14. Binks, B. P. \& Clint, J. H. Solid wettability from surface energy components: Relevance to pickering emulsions. Langmuir 18, 1270-1273 (2002).

15. Binks, B. P. \& Lumsdon, S. O. Pickering emulsions stabilized by monodisperse latex particles: Effects of particle size. Langmuir 17, 4540-4547 (2001)

16. Oettel, M. \& Dietrich, S. Colloidal interactions at fluid interfaces. Langmuir 24, 1425-1441 (2008)

17. Dickinson, E. Adsorbed protein layers at fluid interfaces: interactions, structure and surface rheology. Colloids Surf. B-Biointerfaces 15, 161-176 (1999).

18. Ash, P. A., Bain, C. D. \& Matsubara, H. Wetting in oil/water/surfactant systems. Curr. Opin. Colloid Interface Sci. 17, 196-204 (2012).

19. Nikolaides, M. G. et al. Electric-field-induced capillary attraction between likecharged particles at liquid interfaces. Nature 420, 299-301 (2002).

20. Pieranski, P. Two-dimensional interfacial colloidal crystals. Phys. Rev. Lett. 45 569-572 (1980).

21. Park, B. J. \& Furst, E. M. Attractive interactions between colloids at the oil-water interface. Soft Matter 7, 7676-7682 (2011).

22. Kaz, D. M., McGorty, R., Mani, M., Brenner, M. P. \& Manoharan, V. N. Physical ageing of the contact line on colloidal particles at liquid interfaces. Nat. Mater. 11, 138-142 (2012).

23. Aveyard, R., Clint, J. H., Nees, D. \& Paunov, V. N. Compression and structure of monolayers of charged latex particles at air/water and octane/water interfaces. Langmuir 16, 1969-1979 (2000).

24. Aveyard, R. et al. Measurement of long-range repulsive forces between charged particles at an oil-water interface. Phys. Rev. Lett. 88, (2002).
25. Park, B. J. et al. Direct measurements of the effects of salt and surfactant on interaction forces between colloidal particles at water-oil interfaces. Langmuir 24, 1686-1694 (2008).

26. Park, B. J., Vermant, J. \& Furst, E. M. Heterogeneity of the electrostatic repulsion between colloids at the oil-water interface. Soft Matter 6, 5327-5333 (2010).

27. Maestro, A., Bonales, L. J., Ritacco, H., Rubio, R. G. \& Ortega, F. Effect of the spreading solvent on the three-phase contact angle of microparticles attached at fluid interfaces. Phys. Chem. Chem. Phys. 12, 14115-14120 (2010).

28. Behrens, S. H. \& Grier, D. G. Pair interaction of charged colloidal spheres near a charged wall. Phys. Rev. E 64, (2001).

29. Boneva, M. P., Danov, K. D., Christov, N. C. \& Kralchevsky, P. A. Attraction between particles at a liquid interface due to the interplay of gravity- and electricfield-induced interfacial deformations. Langmuir 25, 9129-9139 (2009).

30. Chen, W., Tan, S. S., Ng, T. K., Ford, W. T. \& Tong, P. Long-ranged attraction between charged polystyrene spheres at aqueous interfaces. Phys. Rev. Lett. 95, (2005).

31. Chen, W. et al. Measured long-ranged attractive interaction between charged polystyrene latex spheres at a water-air interface. Phys. Rev. E 74, (2006).

32. Danov, K. D., Kralchevsky, P. A. \& Boneva, M. P. Electrodipping force acting on solid particles at a fluid interface. Langmuir 20, 6139-6151 (2004).

33. Wang, H. Z., Singh, V. \& Behrens, S. H. Image charge effects on the formation of pickering emulsions. J. Phys. Chem. Lett. 3, 2986-2990 (2012).

34. Singh, P., Joseph, D. D., Gurupatham, S. K., Dalal, B. \& Nudurupati, S. Spontaneous dispersion of particles on liquid surfaces. Proc. Natl. Acad. Sci.USA 106, 19761-19764 (2009)

35. Scriven, L. E. \& Sternling, C. V. Marangoni effects. Nature 187, 186-188 (1960).

36. Lowell, J. \& Truscott, W. S. Triboelectrification of identical insulators 2 theory and further experiments. J. Phys. D-Appl. Phys. 19, 1281-1298 (1986).

37. Lacks, D. J. \& Sankaran, R. M. Contact electrification of insulating materials. J. Phys. D-Appl. Phys. 44, 453001 (2011).

38. Lowell, J. \& Truscott, W. S. Triboelectrification of identical insulators 1 an experimental investigation. J. Phys. D-Appl. Phys. 19, 1273-1280 (1986).

39. Jin, F., Conrad, J. C., Gibiansky, M. L. \& Wong, G. C. L. Bacteria use type-IV pili to slingshot on surfaces. Proc. Natl. Acad. Sci. USA 108, 12617-12622 (2011).

40. Levich, V. G. \& Krylov, V. S. Surface-tension-driven phenomena. Annu. Rev. Fluid. Mech. 1, 293-316 (1969).

41. Nikolov, A. D. et al. Superspreading driven by Marangoni flow. Adv. Colloid. Interface. Sci. 96, 325-338 (2002).

42. Vazquez, G., Alvarez, E. \& Navaza, J. M. Surface-tension of alcohol plus water from 20-degrees-c to 50-degrees-c. J. Chem. Eng. Data 40, 611-614 (1995).

43. Diaz, A. F. \& Felix-Navarro, R. M. A semi-quantitative tribo-electric series for polymeric materials: the influence of chemical structure and properties. J. Electrost. 62, 277-290 (2004).

\section{Acknowledgments}

This work was supported by the National Program on Key Basic Research Project (2012CB933802), the National Natural Science Foundation of China (21104071), (21274141), and the Hong Kong Special Administration Region (HKSAR) General Research Fund (CUHK403210, 2130237).

\section{Author contributions}

P.G. performed the experiments. F.J. and Y.L. analyzed data. X.C.X., T.N. and F.J. contributed jointly to data interpretation and manuscript preparation. All authors reviewed the manuscript.

\section{Additional information}

Supplementary information accompanies this paper at http://www.nature.com/ scientificreports

Competing financial interests: The authors declare no competing financial interests.

How to cite this article: Gao, P., Xing, X.C., Li, Y., Ngai, T. \& Jin, F. Charging and discharging of single colloidal particles at oil/water interfaces. Sci. Rep. 4, 4778; DOI:10.1038/srep04778 (2014).

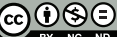

This work is licensed under a Creative Commons Attribution-NonCommercialNoDerivs 3.0 Unported License. The images in this article are included in the article's Creative Commons license, unless indicated otherwise in the image credit; if the image is not included under the Creative Commons license, users will need to obtain permission from the license holder in order to reproduce the image. To view a copy of this license, visit http://creativecommons.org/licenses/by-nc-nd/3.0/ 